\title{
The Master Equation for Two-Level Accelerated Systems at Finite Temperature
}

\author{
J.L. Tomazell:* and R.O. Cunha \\ Departamento de Fisica, Universidade Federal de Santa Catarina, \\ Caixa Postal 476, CEP 88010-970, Florianopolis, S.C., Brasil
}

\begin{abstract}
In this work we study the behaviour of two weakly coupled quantum systems, described by a separable density operator; one of them is a single oscillator, representing a microscopic system, while the other is a set of oscillators which perform the role of a reservoir in thermal equilibrium. From the Liouville-Von Neumann equation for the reduced density operator, we devise the master equation that governs the evolution of the microscopic system, incorporating the effects of temperature via Thermofield Dynamics formalism by suitably redefining the vacuum of the macroscopic system. As applications, we initially investigate the behaviour of a Fermi oscillator in the presence of a heat bath consisting of a set of Fermi oscillators and that of an atomic two-level system interacting with a scalar radiation field, considered as a reservoir, by constructing the corresponding master equation which governs the time evolution of both sub-systems at finite temperature. Finally, we calculate the energy variation rates for the atom and the field, as well as the atomic population levels, both in the inertial case and at constant proper acceleration, considering the two-level system as a prototype of an Unruh detector, for admissible couplings of the radiation field.
\end{abstract}

PACS numbers: 04.62.+v, 42.50.Lc, 12.20.Ds

\footnotetext{
* jeferson.tomazelli@ufsc.br

$\dagger$ renan.oliveira@pgfsc.ufsc.br
} 


\section{INTRODUCTION}

The interest in the study of quantum many-body systems from the modern standpoint of quantum field theories [1-4] has grown since the 1960's, ranging from low to extremely high energy and temperature regimes 5]9]. In 1975, Takahashi and Umezawa proposed an operator-based formalism called Thermofield Dynamics (TFD) [10] in order to describe quantum systems at finite temperature in thermal equilibrium.

In the same year the discovery of the Hawking effect [11, 12], from the black hole thermal radiation, was one of the most innovative results in quantum field theory in curved space-time. Later on, Davis [13] suggested that a similar effect would occur even in a flat space-time. In 1976, Unruh [14] showed that the essential characteristics of the Hawking effect were contained in a simpler situation: an accelerated detector, would be excited by particles in vacuum, property known as the Unruh effect. This discovery of the Hawking-Unruh effect has inspired many researchers, generating since them a series of important results, [15 17]. There are two traditional approaches to the Unruh effect, one is through the quantization of non-massive field in curvilinear coordinates, the other, due to DeWitt [18] and which we shall use in this work, is considering the acceleration as a hyperbolic parametrization in Minkowski space time.

The present work aims at investigating the manifestation of the Unruh effect, approaching the issue from yet another perspective [19, 20]. We consider a two-level system in interaction with a non-massive scalar field, for which we deduce a master equation from Von Neumann equation. Subsequently, we present the thermal states of Thermofield Dynamics (TFD) in the number representation and use it to thermalize the theory via vacuum expected values, singling out in a clearer way the Unruh temperature.

The original proposal of Unruh and DeWitt, considers a detector as a point object that is linearly coupled with a zero mass scalar field through an interaction of monopole type

$$
H_{I}(\tau)=\mu M(\tau) \phi(t(\tau), \mathbf{x}(\tau))
$$

where $\mu$ is a coupling constant and $M(\tau)$ is the monopole moment of the detector. Specifically, $H_{D}$ is the Hamiltonian of the detector which has a discrete spectrum $\left\{E_{i}\right\}$. We assume that the detector moves along the world line described by the coordinate $x(\tau)$, where $\tau$ is the proper time of the detector. Assume that at an initial instant $\tau_{0}$ the detector is in the state $\left|E_{0}\right\rangle$ and the field in the vacuum $|0\rangle$. For an arbitrary trajectory, it is reasonable to assume that the detector will not remain in the initial state, but undergoes a transition to an excited state. Therefore, for a later time $\tau>\tau_{0}$, the detector is in the state $\left|E_{i}\right\rangle$ and field in the state $|\Psi\rangle$. For $\mu$ sufficiently small, we can calculate via first order perturbation theory the transition probability

$$
\mathcal{P}_{t}=\mu^{2} \sum_{i}\left|\left\langle E_{i}|M(0)| E_{0}\right\rangle\right|^{2} \int_{-\tau+\tau_{0}}^{\tau-\tau_{0}} d u e^{-i u \Delta E}\langle 0|\phi(u) \phi(0)| 0\rangle .
$$

The Wightman function in the integrand is well known and can be explicitly calculated, giving

$$
\left\langle 0\left|\phi(t, \mathbf{x}) \phi\left(t^{\prime}, \mathbf{x}^{\prime}\right)\right| 0\right\rangle=\frac{1}{4 \pi^{2}\left[\left|\mathbf{x}\left(\tau^{\prime}\right)-\mathbf{x}\left(\tau^{\prime \prime}\right)\right|^{2}-\left(t\left(\tau^{\prime}\right)-t\left(\tau^{\prime \prime}\right)-i \epsilon\right)^{2}\right]}
$$

Firstly, we consider the case in which the detector follows a inertial world line, i.e., the parametric equations

$$
\begin{gathered}
t(\tau)=\gamma \tau \\
\mathbf{x}(\tau)=\mathbf{x}_{0}+v \gamma \tau,
\end{gathered}
$$

from which we obtain for the Wightman function (3),

$$
\left\langle 0\left|\phi(t, \mathbf{x}) \phi\left(t^{\prime}, \mathbf{x}^{\prime}\right)\right| 0\right\rangle=-\frac{1}{4 \pi^{2}\left(\tau^{\prime}-\tau^{\prime \prime}-i \epsilon^{\prime}\right)^{2}+\mathcal{O}\left(\epsilon^{2}\right)}
$$

and so the probability 


$$
\mathcal{P}_{t}=-\frac{\mu^{2}}{4 \pi^{2}} \sum_{i}\left|\left\langle E_{i}|M(0)| E_{0}\right\rangle\right|^{2} \int_{-\infty}^{\infty} d u \frac{e^{-i u \Delta E}}{\left(u-i \epsilon^{\prime}\right)^{2}}
$$

The integral in (6) turns out to be $\mathcal{P}_{t}=0$. Therefore, the transition probability to an excited state is zero, since, as expected, a detector at rest must not spontaneously excite. Now, consider the case where the detector is uniformly accelerated; this is equivalent to a hyperbolic world line, parametrized according to

$$
\begin{aligned}
& x(\tau)=\alpha^{-1} \cosh (\alpha \tau), \\
& t(\tau)=\alpha^{-1} \sinh (\alpha \tau) .
\end{aligned}
$$

Similarly, for the Wightman function,

$$
\left\langle 0\left|\phi(\tau) \phi\left(\tau^{\prime}\right)\right| 0\right\rangle=-\frac{1}{4 \pi^{2}} \sum_{n=-\infty}^{\infty} \frac{1}{\left[\left(\tau-\tau^{\prime}\right)-i 2 \epsilon+i \frac{2 \pi}{\alpha} n\right]^{2}},
$$

so that the resulting probability is

$$
\mathcal{P}_{t}=-\frac{\mu^{2}}{4 \pi^{2}} \sum_{i}\left|\left\langle E_{i}|M(0)| E_{0}\right\rangle\right|^{2} \sum_{n=-\infty}^{\infty} \int_{-\infty}^{\infty} d u \frac{e^{-i u \Delta E}}{\left[u-i 2 \epsilon+i \frac{2 \pi}{\alpha} n\right]^{2}} .
$$

After integration, it turns out to be

$$
\mathcal{P}_{t}=\frac{\mu^{2}}{2 \pi} \sum_{i}\left|\left\langle E_{i}|M(0)| E_{0}\right\rangle\right|^{2} \frac{\Delta E}{e^{\frac{2 \pi \Delta E}{\alpha}}-1}
$$

from which one recognizes the factor $\left[e^{\frac{2 \pi \Delta E}{\alpha}}-1\right]^{-1}$ as being the Planck distribution associated with the Unruh temperature

$$
T=\frac{\alpha}{2 \pi k_{B}}
$$

where $k_{B}$ is the Boltzmann constant.

\section{THERMOFIELD DYNAMICS}

In this section we briefly present the formalism introduced by Takahashi and Umezawa [4] for a real time thermal field theory, where statistical averages of physical observables correspond to the expected values in a thermal Bogoliubov vacuum.

In Schwinger's Measurement Algebra (SMA) an operator is defined as

$$
X=\sum_{n, m} M(n) X M(m)
$$

where $M(n)=|n\rangle\langle n|$ is known as the measurement symbol or projector into the $n$ basis; defining the composition rule

$$
M(n) M(m)=\delta(n, m) M(m),
$$


in such a context, the expectation value of a given observable $A$ in the single $m$ basis is the scalar

$$
\langle A\rangle_{m}=\operatorname{Tr}[A M(m)]
$$

From that one, we can define the statistical average

$$
\langle A\rangle=\operatorname{Tr}[\rho A]=\sum_{m} \pi(m)\langle A\rangle_{m}
$$

where $\pi(m)$ are the statistical weights and we can recognized the density operator in the SMA as

$$
\rho \equiv \sum_{m} \pi(m) M(m)
$$

It is known that the inclusion of temperature may cause the duplication of the system degrees of freedom [10]. We can achieve this by the introduction of the relation

$$
\delta(n, m)=\delta(\widetilde{n}, \widetilde{m})
$$

once this is done, we can rewrite the density operator as

$$
\begin{gathered}
\rho=\sum_{n, m} \sqrt{\pi(n) \pi(m)} \delta(n, m) M(n, m)=\sum_{n, m} \sqrt{\pi(n) \pi(m)} M(n, \widetilde{n}) M(\widetilde{m}, m) \\
=\left[\sum_{n} \sqrt{\pi(n)} M(n, \tilde{n})\right]\left[\sum_{m} \sqrt{\pi(m)} M(\widetilde{m}, m)\right] \equiv\left|0_{(\beta)}\right\rangle\left\langle 0_{(\beta)}\right|,
\end{gathered}
$$

where we have made use of the composition law of composite measurement symbols. In thermal equilibrium, the statistical weights assume the form $\pi(m)=Z^{-1} e^{-\beta E_{m}}$. In the above expression, we can interpret the density operator as a projector into a thermal vacuum state $\left|0_{(\beta)}\right\rangle$.

For a quantum system in thermal equilibrium at temperature $T$, in the canonical ensemble, the statistical average of an observable $\mathrm{A}$ is then given by

$$
\langle A\rangle \equiv Z^{-1} \operatorname{Tr}[\rho A]=\left\langle 0_{(\beta)}|A| 0_{(\beta)}\right\rangle
$$

where

$$
\begin{gathered}
\left|0_{(\beta)}\right\rangle=Z^{-\frac{1}{2}} \sum_{n} e^{-\frac{1}{2} \beta E_{n}}|n, \widetilde{n}\rangle, \\
|n, \widetilde{n}\rangle \equiv|n\rangle \otimes|\widetilde{n}\rangle .
\end{gathered}
$$

In order to construct this representation it's appropriated to introduce a fictitious system identical to the original system, so that $|\widetilde{n}\rangle$ belongs to this new system. For the free scalar field, we have

$$
\left|0_{(\beta)}\right\rangle_{\mathbf{k}}=\left[1-e^{-\beta \omega_{\mathbf{k}}}\right]^{\frac{1}{2}} \sum_{\mathbf{k}=0}^{\infty} e^{-\frac{1}{2} \beta \omega_{\mathbf{k}} n_{\mathbf{k}}}\left|n_{\mathbf{k}}, \widetilde{n}_{\mathbf{k}}\right\rangle
$$

from which follows the Bose-Einstein distribution

$$
\left\langle 0_{(\beta)}\left|N_{\mathbf{k}}\right| 0_{(\beta)}\right\rangle=\left[e^{\beta \omega_{\mathbf{k}}}-1\right]^{-1}
$$




\section{THE MASTER EQUATION}

In this section we outline the steps leading to the finite temperature master equation following closely the procedure presented in [21] (see also [22 25]). Consider a microscopic system $A$ surrounded by a dissipative environment $R$. The typical Hamiltonian that describes the total system, in the Schrdinger representation, is

$$
H^{S}=H_{A}^{S}+H_{R}^{S}+V^{S}
$$

where $H_{A}^{S}$ is the free Hamiltonian for the system $A, H_{R}^{S}$ corresponds to that for the reservoir $R$ and $V^{S}$ is the interacting potential. Using the density operator formalism to describe the total system, the dynamical evolution, in the interaction representation, will be given by the Liouville-Von Neumann equation,

$$
\frac{d}{d t} \rho(t)=\frac{1}{i \hbar}[V(t), \rho(t)]
$$

Iterating the Liouville-Von Neumann equation to second order, gives

$$
\rho(t+\Delta t)=\rho(t)+\frac{1}{i \hbar} \int_{t}^{t+\Delta t} d t^{\prime}\left[V\left(t^{\prime}\right), \rho(t)\right]+\left(\frac{1}{i \hbar}\right)^{2} \int_{t}^{t+\Delta t} d t^{\prime} \int_{t}^{t^{\prime}} d t^{\prime \prime}\left[V\left(t^{\prime}\right),\left[V\left(t^{\prime \prime}\right), \rho\left(t^{\prime \prime}\right)\right]\right] .
$$

Assuming that the interacting potential is sufficiently weak, the perturbation caused in the reservoir by the microscopic system is negligible and we can consider that the reservoir is in the stationary state

$$
\sigma_{R}(t) \simeq \sigma_{R}(0) \equiv \sigma_{R}
$$

where $\sigma_{R}$ is the reduced density operator for the reservoir. Thus, the density operator for the total system assumes the form [21]

$$
\rho(t)=\sigma_{A}(t) \otimes \sigma_{R}(t)+\rho_{c o r}(t) \simeq \sigma_{A}(t) \otimes \sigma_{R}
$$

The resulting master equation for the reduced density operator in the Markov-Born approximation, or equivalently, the coarse-graining approximation for its time evolution is

$$
\frac{\Delta \sigma_{A}(t)}{\Delta t}=\frac{-1}{\hbar^{2} \Delta t} \int_{t}^{t+\Delta t} d t^{\prime} \int_{t}^{t^{\prime}} d t^{\prime \prime} \operatorname{Tr}_{R}\left[V\left(t^{\prime}\right),\left[V\left(t^{\prime \prime}\right), \sigma_{A}(t) \otimes \sigma_{R}\right]\right]
$$

Assuming a separable potential

$$
V(t)=-R(t) \otimes A(t),
$$

and expanding the master equation commutator we can identify the function

$$
g(t) \equiv \operatorname{Tr}\left[\sigma_{R} R\left(t^{\prime}\right) R\left(t^{\prime \prime}\right)\right]
$$

This is the two-point correlation function and we will use it to introduce temperature in the theory by making the substitution

$$
\sigma_{R}(t) \rightarrow \sigma_{R}(t, \beta)
$$

so that $(30)$ becomes

$$
g(t, \beta)=\left\langle 0_{(\beta)}\left|R\left(t^{\prime}\right) R\left(t^{\prime \prime}\right)\right| 0_{(\beta)}\right\rangle .
$$

Finally, we can derive the finite temperature master equation in the coarse-graining approximation,

$$
\begin{aligned}
\Delta \sigma_{A}(t) & \simeq\left(\frac{1}{i \hbar}\right)^{2} \int_{t}^{t+\Delta t} d t^{\prime} \int_{t}^{t^{\prime}} d t^{\prime \prime} g(t, \beta)\left(A\left(t^{\prime}\right) A\left(t^{\prime \prime}\right) \sigma_{A}\left(t^{\prime \prime}\right)-A\left(t^{\prime \prime}\right) \sigma_{A}\left(t^{\prime \prime}\right) A\left(t^{\prime}\right)\right) \\
& +\left(\frac{1}{i \hbar}\right)^{2} \int_{t}^{t+\Delta t} d t^{\prime} \int_{t}^{t^{\prime}} d t^{\prime \prime} g(-t, \beta)\left(\sigma_{A}\left(t^{\prime \prime}\right) A\left(t^{\prime \prime}\right) A\left(t^{\prime}\right)-A\left(t^{\prime}\right) \sigma_{A}\left(t^{\prime \prime}\right) A\left(t^{\prime \prime}\right)\right)
\end{aligned}
$$




\section{A. The Fermion Oscillator}

We consider the less common case of a Fermi oscillator and how its population, energy levels, $\left\langle n\left|\Delta \sigma_{A}(t)\right| n\right\rangle \equiv$ $\Delta \sigma_{A_{n n}}(t)$ behaves; let the system $A$ be a fermion oscillator of frequency $\omega_{0}$ interacting with a reservoir $R$, composed by $n$ independent oscillators of frequency $\omega_{i}$ in thermal equilibrium. So, we define the system total Hamiltonian by

$$
H=H_{A}+H_{R}+V,
$$

where the Hamiltonian $H$ belongs to the Hilbert space $\mathcal{H}^{1+n}=\mathcal{H} \otimes \mathcal{H}^{n}$, and

$$
\begin{aligned}
& H_{A}=\hbar \omega_{0}\left(b^{S \dagger} b^{S}-\frac{1}{2}\right) \otimes \mathbb{I}, \\
& H_{R}=\mathbb{I} \otimes \hbar \sum_{i} \omega_{i}\left(a_{i}^{S \dagger} a_{i}^{S}-\frac{1}{2}\right),
\end{aligned}
$$

where the respective ladder operators obey the usual anticommuting algebra,

$$
\begin{aligned}
& \left\{b, b^{\dagger}\right\}=1 \\
& \left\{a_{i}, a_{j}\right\}=\delta_{i, j} .
\end{aligned}
$$

In order to describe the interaction with the reservoir, we introduce the potential

$$
V=\sum_{i}\left[g_{i}\left(\epsilon b^{S}+\eta b^{S \dagger}\right) a_{i}^{S}+g_{i}^{*} a_{i}^{S \dagger}\left(\epsilon b^{S \dagger}+\eta b^{S}\right)\right]
$$

where $g_{i}$ is a coupling parameter and $\epsilon, \eta$ non-negative constants, the expression above being the most general sesquilinear interaction. Making $\eta=1$ and assuming the rotating wave approximation, $\epsilon=0$, we arrive at

$$
V=-\left(b^{S \dagger} \otimes R^{S}+b^{S} \otimes R^{S \dagger}\right),
$$

where

$$
R^{S}=-\left(g_{1} a_{1}^{S} \otimes \mathbb{I} \otimes \mathbb{I} \cdots+\mathbb{I} \otimes g_{2} a_{2}^{S} \otimes \mathbb{I} \cdots+\cdots\right)=-\sum_{i} g_{i} a_{i}^{S} .
$$

Writing the master equation commutator explicitly, we have

$$
\begin{aligned}
& \operatorname{Tr}_{R}\left[V\left(t^{\prime}\right),\left[V\left(t^{\prime \prime}\right), \sigma_{A}(t) \otimes \sigma_{R}\right]\right] \\
& =\left(b\left(t^{\prime}\right) b^{\dagger}\left(t^{\prime \prime}\right) \sigma_{A}(t)-b^{\dagger}\left(t^{\prime \prime}\right) \sigma_{A}(t) b\left(t^{\prime}\right)\right) \operatorname{Tr}\left[\sigma_{R} R^{\dagger}\left(t^{\prime}\right) R\left(t^{\prime \prime}\right)\right] \\
& +\left(\sigma_{A}(t) b\left(t^{\prime \prime}\right) b^{\dagger}\left(t^{\prime}\right)-b^{\dagger}\left(t^{\prime}\right) \sigma_{A}(t) b\left(t^{\prime \prime}\right)\right) \operatorname{Tr}\left[\sigma_{R} R^{\dagger}\left(t^{\prime \prime}\right) R\left(t^{\prime}\right)\right] \\
& +\left(b^{\dagger}\left(t^{\prime}\right) b\left(t^{\prime \prime}\right) \sigma_{A}(t)-b\left(t^{\prime \prime}\right) \sigma_{A}(t) b^{\dagger}\left(t^{\prime}\right)\right) \operatorname{Tr}\left[\sigma_{R} R\left(t^{\prime}\right) R^{\dagger}\left(t^{\prime \prime}\right)\right] \\
& +\left(\sigma_{A}(t) b^{\dagger}\left(t^{\prime \prime}\right) b\left(t^{\prime}\right)-b\left(t^{\prime}\right) \sigma_{A}(t) b^{\dagger}\left(t^{\prime \prime}\right)\right) \operatorname{Tr}\left[\sigma_{R} R\left(t^{\prime \prime}\right) R^{\dagger}\left(t^{\prime}\right)\right] .
\end{aligned}
$$

By ensuring that the vacuum of the system is separable

$$
\left|0_{(\beta)}\right\rangle=\left|0_{(\beta)}\right\rangle_{1} \otimes\left|0_{(\beta)}\right\rangle_{2} \otimes \cdots,
$$

we also guarantee the separability of the function $g(t, \beta)$,

$$
\begin{gathered}
\operatorname{Tr}\left[\sigma_{R} R\left(t^{\prime}\right) R\left(t^{\prime \prime}\right)\right]=\left\langle 0_{(\beta)}\left|R\left(t^{\prime}\right) R\left(t^{\prime \prime}\right)\right| 0_{(\beta)}\right\rangle \\
=\left\langle 0_{(\beta)}\left|R\left(t^{\prime}\right) R\left(t^{\prime \prime}\right)\right| 0_{(\beta)}\right\rangle_{1}\left\langle 0_{(\beta)}\left|R\left(t^{\prime}\right) R\left(t^{\prime \prime}\right)\right| 0_{(\beta)}\right\rangle_{2} \cdots .
\end{gathered}
$$


The fermion traces follow from the above expression, in which there appears, as expected, the Fermi-Dirac distribution,

$$
\begin{aligned}
& \operatorname{Tr}\left[\sigma_{R} R^{\dagger}\left(t^{\prime}\right) R\left(t^{\prime \prime}\right)\right]=\sum_{i}\left|g_{i}\right|^{2} e^{-i \omega_{i}\left(t^{\prime \prime}-t^{\prime}\right)}\left(e^{\beta \hbar \omega}+1\right)^{-1}, \\
& \operatorname{Tr}\left[\sigma_{R} R^{\dagger}\left(t^{\prime \prime}\right) R\left(t^{\prime}\right)\right]=\sum_{i}\left|g_{i}\right|^{2} e^{-i \omega_{i}\left(t^{\prime}-t^{\prime \prime}\right)}\left(e^{\beta \hbar \omega}+1\right)^{-1}, \\
& \operatorname{Tr}\left[\sigma_{R} R\left(t^{\prime}\right) R^{\dagger}\left(t^{\prime \prime}\right)\right]=\sum_{i}\left|g_{i}\right|^{2} e^{-i \omega_{i}\left(t^{\prime}-t^{\prime \prime}\right)}\left[1-\left(e^{\beta \hbar \omega}+1\right)^{-1}\right], \\
& \operatorname{Tr}\left[\sigma_{R} R\left(t^{\prime \prime}\right) R^{\dagger}\left(t^{\prime}\right)\right]=\sum_{i}\left|g_{i}\right|^{2} e^{-i \omega_{i}\left(t^{\prime \prime}-t^{\prime}\right)}\left[1-\left(e^{\beta \hbar \omega}+1\right)^{-1}\right] .
\end{aligned}
$$

Under these considerations, we arrive at the equation for the evolution of the "population of a given state $n$, for the microscopic system,

$$
\begin{gathered}
\frac{\Delta \sigma_{A_{n n}}(t)}{\Delta t}=-n C \sigma_{A_{n n}}(t)+(n+1) C \sigma_{A_{n+1, n+1}}(t) \\
-(n+1) T_{F}\left(\sigma_{A_{n+1, n+1}}(t)+\sigma_{A_{n n}}(t)\right)+n T_{F}\left(\sigma_{A_{n-1, n-1}}(t)+\sigma_{A_{n n}}(t)\right),
\end{gathered}
$$

where

$$
\begin{aligned}
C & \equiv 2\left(\frac{1}{\hbar}\right)^{2} \sum_{i}\left|g_{i}\right|^{2} \omega_{0 i}^{-2}\left[\frac{1-\cos \left(\omega_{0 i} \Delta t\right)}{\Delta t}\right] \\
T_{F} & \equiv 2\left(\frac{1}{\hbar}\right)^{2} \sum_{i}\left|g_{i}\right|^{2} \omega_{0 i}^{-2}\left[\frac{1-\cos \left(\omega_{0 i} \Delta t\right)}{\Delta t}\right]\left(e^{\beta \hbar \omega_{i}}+1\right)^{-1} .
\end{aligned}
$$

Interpreting the above equation, we realize that $C$ is associated to the spontaneous emission and $T_{F}$ to the process of stimulated absorption and emission. More precisely, $n C$ is the spontaneous emission rate between the states $|n\rangle$ and $|n+1\rangle$, so the state $|n\rangle$ decays at a rate $n C$ while the state $|n+1\rangle$ is populated at rate $(n+1) C$. Similarly, there are processes of absorption and stimulated emission, at rates $n T_{F}$ and $(n+1) T_{F}$, respectively. Observing the thermodynamic limit of transition rates for low temperatures,

$$
\beta E \gg 1, T_{F} \rightarrow 0
$$

we find that the only allowed variations are the fluctuations. However, in the high temperatures limit,

$$
\beta E \ll 1, T_{F} \rightarrow \frac{1}{2} C .
$$

The transition rate takes the value $\frac{1}{2}$, due to restrictions imposed by the fermion algebra, allowing the excitation of the only mode of energy available. At progressively higher temperatures, the fermion particles of the thermal bath exchange energy with the microscopic particle through short-range correlations, exciting it or inducing it to decay to their only two modes of oscillation, with equal probabilities. Finally, if we want to describe a boson oscillator in a fermion heat bath we use the expression without restricting the accessible states.

In the fermion case having only two allowed modes we can easily see how the energy for the microscopic system evolves

$$
\begin{gathered}
\frac{d}{d t}\left\langle H_{A}\right\rangle_{A}=\frac{d}{d t} \operatorname{Tr}\left[\sigma_{A}(t) H_{A}\right]=\operatorname{Tr}\left[\left(\frac{d}{d t} \sigma_{A}(t)\right) H_{A}\right] \\
=\sum_{n}\left\langle n\left|\left(\frac{d}{d t} \sigma_{A}(t)\right) H_{A}\right| n\right\rangle=\sum_{n} \hbar \omega_{0}\left(n-\frac{1}{2}\right)\left\langle n\left|\left(\frac{d}{d t} \sigma_{A}(t)\right)\right| n\right\rangle \\
=-\hbar \omega_{0} \frac{1}{2}\left\langle 0\left|\left(\frac{d}{d t} \sigma_{A}(t)\right)\right| 0\right\rangle+\hbar \omega_{0} \frac{1}{2}\left\langle 1\left|\left(\frac{d}{d t} \sigma_{A}(t)\right)\right| 1\right\rangle \\
=\hbar \omega_{0}\left(-C \sigma_{A_{1,1}}(t)+T_{F} \sigma_{A_{00}}(t)\right) .
\end{gathered}
$$


If we look for a stationary state

$$
\frac{d}{d t}\left\langle H_{A}\right\rangle_{A}=0
$$

we find that we can only reach such state with $T=0 K$, so the microscopic system energy stays oscillating in a local non equilibrium situation; this is fundamental and enables us to construct the master equation.

\section{B. Master equation for a two-level system}

In order to go further and integrate the equation (33), it is necessary to know the form of $\sigma_{A}\left(t^{\prime \prime}\right)$. For this reason, we propose a two-level atomic system described by the Hamiltonian

$$
H_{A}=\omega_{0} R_{3}(\tau)
$$

where $R_{3}=\frac{1}{2}|+\rangle\left\langle+\left|-\frac{1}{2}\right|-\right\rangle\langle-|$ and $\tau$ is the proper time of the atom, whose eigenvalues are

$$
\begin{aligned}
& H_{A}|+\rangle=\frac{1}{2} \omega_{0}|+\rangle, \\
& H_{A}|-\rangle=-\frac{1}{2} \omega_{0}|-\rangle .
\end{aligned}
$$

The proposed field-atom interaction is similar to (11) and given by the Hamiltonian

$$
H_{I}=\mu R_{2}(\tau) \phi(\tau)
$$

where $\mu$ is the coupling constant and $R_{2}=\frac{i}{2}\left(R_{-}-R_{+}\right)$, with $R_{+}=\frac{i}{2}|+\rangle\langle-|$ and $R_{-}=\frac{i}{2}|-\rangle\langle+|$. It is noteworthy that the operators $R_{+}$and $R_{-}$can be seen as raising and lowering operators of the atomic system and, together with $R_{3}$, generate the $S O(3)$ algebra,

$$
\left[R_{+}, R_{-}\right]=2 R_{3}
$$

$$
\left[R_{3}, R_{ \pm}\right]= \pm R_{ \pm}
$$

From the Heisenberg equation

$$
i \frac{d}{d t} A(t)=\left[A(t), H_{T}\right]
$$

where $H_{T}=H_{A}+H_{F}+H_{I}$, we obtain the evolution equations

$$
\begin{gathered}
\frac{d}{d \tau} R_{ \pm}(\tau)= \pm i \omega_{0} R_{ \pm}(\tau)+i \mu \phi(\tau)\left[R_{2}(\tau), R_{ \pm}(\tau)\right] \\
\frac{d}{d \tau} R_{3}(\tau)=i \mu \phi(\tau)\left[R_{2}(\tau), R_{3}(\tau)\right]
\end{gathered}
$$

Working on (54) and (55) to first order in $\mu$ we can conveniently breaking then down into a free part, which preserves in the absence of coupling,

$$
R_{ \pm}^{f}(\tau)=R_{ \pm}^{f}\left(\tau_{0}\right) e^{ \pm i \omega_{0}\left(\tau-\tau_{0}\right)}
$$




$$
R_{3}^{f}(\tau)=R_{3}\left(\tau_{0}\right)
$$

and another part of the resulting coupling

$$
\begin{aligned}
& R_{ \pm}^{S}(\tau)=i \mu \int_{\tau_{0}}^{\tau} d \tau^{\prime} \phi^{f}\left(\tau^{\prime}\right)\left[R_{2}^{f}\left(\tau^{\prime}\right), R_{ \pm}^{f}(\tau)\right] \\
& R_{3}^{S}(\tau)=i \mu \int_{\tau_{0}}^{\tau} d \tau^{\prime} \phi^{f}\left(\tau^{\prime}\right)\left[R_{2}^{f}\left(\tau^{\prime}\right), R_{3}^{f}(\tau)\right]
\end{aligned}
$$

where $\phi^{f}(\tau)$ is the free part of the field, $\phi(\tau)=\phi^{f}(\tau)+\phi^{S}(\tau)$,

$$
\begin{gathered}
\phi^{f}(\tau)=(2 \pi)^{-3 / 2} \int \frac{d^{3} \mathrm{k}}{\sqrt{2 \omega_{\mathbf{k}}}}\left[a_{\mathbf{k}} e^{-i\left(\omega_{\mathbf{k}} t-\mathbf{k} \cdot \mathbf{x}\right)}+a_{\mathbf{k}}^{\dagger} e^{i\left(\omega_{\mathbf{k}} t-\mathbf{k} \cdot \mathbf{x}\right)}\right], \\
\phi^{S}(\tau)=i \mu \int_{\tau_{0}}^{\tau} d \tau^{\prime} R_{2}^{f}\left(\tau^{\prime}\right)\left[\phi^{f}\left(\tau^{\prime}\right), \phi^{f}(\tau)\right] .
\end{gathered}
$$

Therefore, we write the atom energy variation, $H_{A}(\tau)$, as

$$
\frac{d}{d \tau} H_{A}(\tau)=-\mu \phi(\tau) \frac{d}{d \tau} R_{2}^{f}(\tau)-i \mu^{2} \int_{\tau_{0}}^{\tau} d \tau^{\prime} \phi(\tau) \phi^{f}\left(\tau^{\prime}\right) \frac{d}{d \tau}\left[R_{2}^{f}\left(\tau^{\prime}\right), R_{2}^{f}(\tau)\right] .
$$

One of the purpose of this section is to identify and quantitatively analyze the contributions from the vacuum fluctuations and radiation reaction, so, we can be identified that the free part of the filed (60) is related to the vacuum fluctuations and, consequently, the radiation reaction is caused by the interaction part (61). The energy is a physical observable and, as such, represented by a hermitian operator, we would like to recognize these two different contributions on the energy variation and associates them with two physical processes which are represented by hermitian operators. For this condition to be valid it is necessary to perform the ordering of operators in (62) - Acknowledging that in quantum theory exists one indeterminacy as to the order of operators, lets consider the product $\lambda A B+(1-\lambda) B A$, resulting for the vacuum fluctuation

$$
\begin{gathered}
\left(\frac{d}{d \tau} H_{A}(\tau)\right)_{V F}=\lambda\left\{-\mu \phi^{f}(\tau) \frac{d}{d \tau} R_{2}^{f}(\tau)-i \mu^{2} \int_{\tau_{0}}^{\tau} d \tau^{\prime} \phi^{f}(\tau) \phi^{f}\left(\tau^{\prime}\right) \frac{d}{d \tau}\left[R_{2}^{f}\left(\tau^{\prime}\right), R_{2}^{f}(\tau)\right]\right\} \\
+(1-\lambda)\left\{-\mu \frac{d}{d \tau} R_{2}^{f}(\tau) \phi^{f}(\tau)-i \mu^{2} \int_{\tau_{0}}^{\tau} d \tau^{\prime} \phi^{f}\left(\tau^{\prime}\right) \phi^{f}(\tau) \frac{d}{d \tau}\left[R_{2}^{f}\left(\tau^{\prime}\right), R_{2}^{f}(\tau)\right]\right\}
\end{gathered}
$$

and for the radiation reaction

$$
\left(\frac{d}{d \tau} H_{A}(\tau)\right)_{R R}=-i \mu^{2} \int_{\tau_{0}}^{\tau} d \tau^{\prime}\left[\phi^{f}\left(\tau^{\prime}\right), \phi^{f}(\tau)\right]\left[\lambda R_{2}^{f}\left(\tau^{\prime}\right) \frac{d}{d \tau} R_{2}^{f}(\tau)+(1-\lambda)\left(\frac{d}{d \tau} R_{2}^{f}(\tau)\right) R_{2}^{f}\left(\tau^{\prime}\right)\right] .
$$

Taking the expected value $\left\langle\frac{d}{d \tau} H_{A}(\tau)\right\rangle \equiv\left\langle a\left|\otimes\left\langle 0\left|\frac{d}{d \tau} H_{A}(\tau)\right| 0\right\rangle \otimes\right| a\right\rangle$, where $|0\rangle$ is the vacuum field and $|a\rangle$ an arbitrary atom state, results

$$
\begin{gathered}
\left\langle\frac{d}{d \tau} H_{A}(\tau)\right\rangle_{V F}=i \mu^{2} \int_{\tau_{0}}^{\tau} d \tau^{\prime} \frac{d}{d \tau}\left\langle a\left|\left[R_{2}^{f}(\tau), R_{2}^{f}\left(\tau^{\prime}\right)\right]\right| a\right\rangle \times \\
\left\langle 0\left|\left(\lambda-\frac{1}{2}\right)\left[\phi^{f}(\tau), \phi^{f}\left(\tau^{\prime}\right)\right]+\frac{1}{2}\left[\phi^{f}(\tau), \phi^{f}\left(\tau^{\prime}\right)\right]_{+}\right| 0\right\rangle
\end{gathered}
$$




$$
\begin{gathered}
\left\langle\frac{d}{d \tau} H_{A}(\tau)\right\rangle_{R R}=i \mu^{2} \int_{\tau_{0}}^{\tau} d \tau^{\prime}\left\langle 0\left|\left[\phi^{f}(\tau), \phi^{f}\left(\tau^{\prime}\right)\right]\right| 0\right\rangle \times \\
\frac{d}{d \tau}\left\langle a\left|\frac{1}{2}\left[R_{2}^{f}(\tau), R_{2}^{f}\left(\tau^{\prime}\right)\right]_{+}-\left(\lambda-\frac{1}{2}\right)\left[R_{2}^{f}(\tau), R_{2}^{f}\left(\tau^{\prime}\right)\right]\right| a\right\rangle .
\end{gathered}
$$

In equations (65) and (66) we can recognize the commonly known functions in quantum optics as correlation function $C\left(\tau, \tau^{\prime}\right)$ and linear susceptibility $\chi\left(\tau, \tau^{\prime}\right)$. For the field, the correlation and susceptibility functions are

$$
\begin{aligned}
C^{F}\left(\tau, \tau^{\prime}\right) & =\frac{1}{2}\left\langle 0\left|\left[\phi^{f}(\tau), \phi^{f}\left(\tau^{\prime}\right)\right]_{+}\right| 0\right\rangle, \\
\chi^{F}\left(\tau, \tau^{\prime}\right) & =\frac{1}{2 i}\left\langle 0\left|\left[\phi^{f}(\tau), \phi^{f}\left(\tau^{\prime}\right)\right]\right| 0\right\rangle,
\end{aligned}
$$

and for the atomic system

$$
\begin{aligned}
& C^{A}\left(\tau, \tau^{\prime}\right)=\frac{1}{2}\left\langle a\left|\left[R_{2}^{f}(\tau), R_{2}^{f}\left(\tau^{\prime}\right)\right]_{+}\right| a\right\rangle, \\
& \chi^{A}\left(\tau, \tau^{\prime}\right)=\frac{1}{2 i}\left\langle a\left|\left[R_{2}^{f}(\tau), R_{2}^{f}\left(\tau^{\prime}\right)\right]\right| a\right\rangle .
\end{aligned}
$$

Explicit calculation of the correlation and susceptibility functions gives, to the atomic system,

$$
\begin{gathered}
C^{A}\left(\tau, \tau^{\prime}\right)=\frac{1}{4} \cos \left(\omega_{0}\left(\tau-\tau^{\prime}\right)\right), \\
\chi^{A}\left(\tau, \tau^{\prime}\right)=\frac{1}{2} \sin \left(\omega_{0}\left(\tau-\tau^{\prime}\right)\right)\left\langle a\left|R_{3}^{f}\left(\tau_{0}\right)\right| a\right\rangle,
\end{gathered}
$$

and, using Wightman functions, to the field

$$
\begin{aligned}
& C^{F}\left(\tau, \tau^{\prime}\right)=\frac{1}{8 \pi^{2}}\left[\frac{1}{\left[|\Delta \mathbf{x}|^{2}-(\Delta t-i \epsilon)^{2}\right]}+\frac{1}{\left[|\Delta \mathbf{x}|^{2}-(\Delta t+i \epsilon)^{2}\right]}\right], \\
& \chi^{F}\left(\tau, \tau^{\prime}\right)=\frac{1}{8 \pi^{2} i}\left[\frac{1}{\left[|\Delta \mathbf{x}|^{2}-(\Delta t-i \epsilon)^{2}\right]}-\frac{1}{\left[|\Delta \mathbf{x}|^{2}-(\Delta t+i \epsilon)^{2}\right]}\right] .
\end{aligned}
$$

To go beyond (73) and (74) one must choose a parametrization, thus, using the uniformly accelerated case as before (7) results

$$
\begin{aligned}
& C^{F}\left(\tau, \tau^{\prime}\right)=-\frac{1}{8 \pi^{2}} \sum_{n=-\infty}^{\infty}\left[\frac{1}{\left(\Delta \tau-i \epsilon 2+i \frac{2 \pi}{\alpha} n\right)^{2}}+\frac{1}{\left(\Delta \tau+i \epsilon 2+i \frac{2 \pi}{\alpha} n\right)^{2}}\right], \\
& \chi^{F}\left(\tau, \tau^{\prime}\right)=-\frac{1}{8 \pi^{2} i} \sum_{n=-\infty}^{\infty}\left[\frac{1}{\left(\Delta \tau-i \epsilon 2+i \frac{2 \pi}{\alpha} n\right)^{2}}-\frac{1}{\left(\Delta \tau+i \epsilon 2+i \frac{2 \pi}{\alpha} n\right)^{2}}\right] .
\end{aligned}
$$


Unfortunately, in this case, the integrals found in (65) and (66) are divergent. One option to overcome this situation is to restrict the study to the asymptotic case, $\tau_{0} \rightarrow-\infty$, leading to

$$
\begin{aligned}
& \left\langle\frac{d}{d \tau} H_{A}(\tau)\right\rangle_{V F}=-\omega_{0} \mu^{2}\left\langle a\left|R_{3}\right| a\right\rangle\left[\frac{\omega_{0}}{8 \pi}\left(1+\frac{2}{e^{\frac{2 \pi \omega_{0}}{\alpha}}-1}\right)+i(2 \lambda-1) \int_{0}^{\infty} d u \cos \left(\omega_{0} u\right) \chi^{F}(u)\right] \\
& \left\langle\frac{d}{d \tau} H_{A}(\tau)\right\rangle_{R R}=\omega_{0} \mu^{2}\left[-\frac{\omega_{0}}{16 \pi}\left(1+\frac{2}{e^{\frac{2 \pi \omega_{0}}{\alpha}}-1}\right)+i(2 \lambda-1)\left\langle a\left|R_{3}\right| a\right\rangle \int_{0}^{\infty} d u \cos \left(\omega_{0} u\right) \chi^{F}(u)\right]
\end{aligned}
$$

where $u=\tau-\tau^{\prime}$. Nevertheless, the integral

$$
\int_{0}^{\infty} d u \cos \left(\omega_{0} u\right) \chi^{F}(u)=-\frac{1}{8 \pi^{2} i} \sum_{n=-\infty}^{\infty} \int_{0}^{\infty} d u \cos \left(\omega_{0} u\right)\left[\frac{1}{\left(u-i \epsilon 2+i \frac{2 \pi}{\alpha} n\right)^{2}}-\frac{1}{\left(u+i \epsilon 2+i \frac{2 \pi}{\alpha} n\right)^{2}}\right]
$$

is still diverging. From equations (77) and (78) can be concluded two important facts. For the physical observable associated with vacuum fluctuations and radiation reaction be independent and represented by finite quantities it is necessary to perform operators ordering. It's also clear that the only possible order to yield not divergent rates is the symmetrical one, $\lambda=\frac{1}{2}$. Thus, for the symmetric case,

$$
\begin{gathered}
\left\langle\frac{d}{d \tau} H_{A}(\tau)\right\rangle_{V F}=-\frac{\omega_{0}^{2} \mu^{2}}{8 \pi}\left\langle a\left|R_{3}\right| a\right\rangle\left[1+\frac{2}{e^{\frac{2 \pi \omega_{0}}{\alpha}}-1}\right], \\
\left\langle\frac{d}{d \tau} H_{A}(\tau)\right\rangle_{R R}=-\frac{\omega_{0}^{2} \mu^{2}}{16 \pi}\left[1+\frac{2}{e^{\frac{2 \pi \omega_{0}}{\alpha}}-1}\right] .
\end{gathered}
$$

From (81), we notice that the radiation reaction is responsible for giving energy to the field and has a purely dissipative nature, independent of $\mu$ and $\omega_{0}$. To better interpret the vacuum fluctuations (80), we replace $R_{3}$ for its explicit form

$$
\left\langle\frac{d}{d \tau} H_{A}(\tau)\right\rangle_{V F}=-\frac{\omega_{0}^{2} \mu^{2}}{16 \pi}\left[1+\frac{2}{e^{\frac{2 \pi \omega_{0}}{\alpha}}-1}\right]\left[|\langle a \mid+\rangle|^{2}-|\langle a \mid-\rangle|^{2}\right],
$$

therefore, in the case where $|a\rangle=|+\rangle$, i.e. the atom is in the excited state, results

$$
\left\langle+\left|\frac{d}{d \tau} H_{A}(\tau)\right|+\right\rangle_{V F}=-\frac{\omega_{0}^{2} \mu^{2}}{16 \pi}\left[1+\frac{2}{e^{\frac{2 \pi \omega_{0}}{\alpha}}-1}\right]
$$

and, as expected, the vacuum fluctuation is responsible for giving energy. In case that $|a\rangle=|-\rangle$, which is equivalent to the ground state,

$$
\left\langle-\left|\frac{d}{d \tau} H_{A}(\tau)\right|-\right\rangle_{V F}=\frac{\omega_{0}^{2} \mu^{2}}{16 \pi}\left[1+\frac{2}{e^{\frac{2 \pi \omega_{0}}{\alpha}}-1}\right]
$$

so the vacuum fluctuation is responsible for excite the atom. Note that, on average, vacuum fluctuations cause a zero energy change, as expected, since it is related to the free part of the field and so taking place regardless of the interaction. Therefore, it is not unusual to expect that the average variation caused by it to be zero.

Returning to equations (77) and (78), observe that when adding both

$$
\left\langle\frac{d}{d \tau} H_{A}(\tau)\right\rangle_{T}=-\frac{\omega_{0}^{2} \mu^{2}}{8 \pi}\left[\frac{1}{2}+\left\langle a\left|R_{3}\right| a\right\rangle\right]\left[1+\frac{2}{e^{\frac{2 \pi \omega_{0}}{\alpha}}-1}\right],
$$


and the equation (85) has no dependence of the parameter $\lambda$, that is, for the total physical process any ordering results in the same energy variation [9].

Keeping this in mind, we now establish the following correspondences between (50) and (29) :

$$
\left\{\begin{array}{l}
t \rightarrow \tau \\
A(t) \rightarrow R_{2}(\tau) \\
R(t) \rightarrow \phi(\tau)
\end{array}\right.
$$

Here, equation (33) is projected in the basis of the eigenstates of the atomic system. Thus, taking the expectation value of equation (33) in an arbitrary state $|a\rangle$ of the atomic system and defined the contracted notation $\Delta \sigma_{A_{a a}}(\tau) \equiv$ $\left\langle a\left|\Delta \sigma_{A}(\tau)\right| a\right\rangle$, equation becomes

$$
\frac{\Delta \sigma_{A_{a a}}(\tau)}{\Delta \tau} \simeq-\frac{1}{4 \Delta \tau} \int_{\tau}^{\tau+\Delta \tau} d \tau^{\prime} \int_{\tau}^{\tau^{\prime}} d \tau^{\prime \prime} g(\tau, \beta)\left[e^{a i \omega_{0}\left(\tau^{\prime}-\tau^{\prime \prime}\right)} \sigma_{A_{a a}}(\tau)-e^{-a i \omega_{0}\left(\tau^{\prime}-\tau^{\prime \prime}\right)} \sigma_{A_{b b}}(\tau)\right]+h . c .
$$

where h.c. represents the hermitian conjugate. Equation (32) is, in this case, $g(\tau, \beta)=\left\langle 0_{(\beta)}\left|\phi\left(\tau^{\prime}\right) \phi\left(\tau^{\prime \prime}\right)\right| 0_{(\beta)}\right\rangle$. Replacing the fields, we obtain

$$
g\left(\tau^{\prime}, \tau^{\prime \prime}\right)=(2 \pi)^{-3} \int \frac{d^{3} \mathrm{k}}{2 \omega_{\mathbf{k}}}\left[\left\langle 0_{(\beta)}\left|N_{\mathbf{k}}\right| 0_{(\beta)}\right\rangle e^{i \omega_{\mathbf{k}}\left(\tau^{\prime}-\tau^{\prime \prime}\right)-i \mathbf{k} \cdot\left(\mathbf{x}^{\prime}-\mathbf{x}^{\prime \prime}\right)}+\left(\left\langle 0_{(\beta)}\left|N_{\mathbf{k}}\right| 0_{(\beta)}\right\rangle+1\right) e^{-i \omega_{\mathbf{k}}\left(\tau^{\prime}-\tau^{\prime \prime}\right)+i \mathbf{k} \cdot\left(\mathbf{x}^{\prime}-\mathbf{x}^{\prime \prime}\right)}\right]
$$

or, using the results found for the expected value of the number operator (22),

$$
g\left(\tau^{\prime}, \tau^{\prime \prime}\right)=(2 \pi)^{-3} \int \frac{d^{3} \mathbf{k}^{\prime}}{2 \omega_{\mathbf{k}}}\left[\left[e^{\beta \omega_{\mathbf{k}}}-1\right]^{-1} e^{i \omega_{\mathbf{k}}\left(\tau^{\prime}-\tau^{\prime \prime}\right)-i \mathbf{k} \cdot\left(\mathbf{x}^{\prime}-\mathbf{x}^{\prime \prime}\right)}+\left[1-e^{-\beta \omega_{\mathbf{k}}}\right]^{-1} e^{-i \omega_{\mathbf{k}}\left(\tau^{\prime}-\tau^{\prime \prime}\right)+i \mathbf{k} \cdot\left(\mathbf{x}^{\prime}-\mathbf{x}^{\prime \prime}\right)}\right] .
$$

Next, consider the master equation in the inertial case and, subsequently, in the case of an accelerated frame. In the inertial case,

$$
\begin{gathered}
g\left(\tau^{\prime}, \tau^{\prime \prime}\right)=\frac{1}{4 \pi^{2}} \sum_{n=-\infty}^{\infty} \frac{1}{i 2 \beta(\gamma-1)\left(\tau^{\prime}-\tau^{\prime \prime}\right)(n+\epsilon)-\left[\left(\tau^{\prime}-\tau^{\prime \prime}\right)-i \beta(n+\epsilon)\right]^{2}} \\
=\frac{\sqrt{1-v^{2}}\left[\operatorname{coth}\left(\frac{(v-1)}{\sqrt{1-v^{2}}} \frac{\pi}{\beta}\left(\tau^{\prime}-\tau^{\prime \prime}\right)\right)+\operatorname{coth}\left(\frac{(v+1)}{\sqrt{1-v^{2}}} \frac{\pi}{\beta}\left(\tau^{\prime}-\tau^{\prime \prime}\right)\right)\right]}{8 \pi \beta v\left(\tau^{\prime}-\tau^{\prime \prime}\right)} .
\end{gathered}
$$

The above expression is too complicated to be integrated. However, in the limit $v \rightarrow 0$,

$$
g\left(\tau^{\prime}, \tau^{\prime \prime}\right)=-\frac{1}{4 \pi^{2}} \sum_{n=-\infty}^{\infty} \frac{1}{\left[\left(\tau^{\prime}-\tau^{\prime \prime}\right)-i \beta(n+\epsilon)\right]^{2}}=-\frac{1}{4 \beta^{2}} \operatorname{csch}\left(\frac{\pi\left(\tau^{\prime}-\tau^{\prime \prime}\right)}{\beta}\right)^{2}
$$

In this case, equation (87) becomes

$$
\frac{\Delta \sigma_{A_{a a}}(\tau)}{\Delta \tau} \simeq \frac{1}{16 \pi^{2}} \sum_{n=-\infty}^{\infty} \int_{-\infty}^{\infty} d u[u-i \beta(n+\epsilon)]^{-2}\left[e^{a i \omega_{0} u} \sigma_{A_{a a}}(\tau)-e^{-a i \omega_{0} u} \sigma_{A_{b b}}(\tau)\right]
$$

Writing the master equation (91) for the states $|+\rangle$ e $|-\rangle$, results

$$
\begin{aligned}
& \frac{\Delta \sigma_{+}(\tau)}{\Delta \tau} \simeq-\frac{\omega_{0}}{8 \pi}\left\{\sigma_{-}(\tau)+\frac{1}{1-e^{-\omega_{0} \beta}}\left[\sigma_{+}(\tau)-\sigma_{-}(\tau)\right]\right\}, \\
& \frac{\Delta \sigma_{-}(\tau)}{\Delta \tau} \simeq \frac{\omega_{0}}{8 \pi}\left\{\sigma_{-}(\tau)+\frac{1}{1-e^{-\omega_{0} \beta}}\left[\sigma_{+}(\tau)-\sigma_{-}(\tau)\right]\right\} .
\end{aligned}
$$


From (92) e (93), it follows that

$$
\frac{\Delta \sigma_{+}(\tau)}{\Delta \tau}=-\frac{\Delta \sigma_{-}(\tau)}{\Delta \tau}
$$

At steady regime, $\frac{\Delta \sigma_{+}(\tau)}{\Delta \tau}=0=\frac{\Delta \sigma_{-}(\tau)}{\Delta \tau}$, we find the detailed balance condition

$$
\frac{\sigma_{+}(\tau)}{\sigma_{-}(\tau)}=e^{-\omega_{0} \beta}
$$

Solving the coupled equations (92) and (93), with the initial conditions $\sigma_{+}(0)=\sigma_{0+}$ e $\sigma_{-}(0)=\sigma_{0-}$ and the boundary conditions $\sigma_{+}(\tau)+\sigma_{-}(\tau)=1$, we find

$$
\begin{gathered}
\sigma_{+}(\tau)=\sigma_{0+} e^{-\operatorname{coth}\left(\frac{1}{2} \omega_{0} \beta\right) \frac{\omega_{0}}{8 \pi} \tau}-\frac{e^{-\operatorname{coth}\left(\frac{1}{2} \omega_{0} \beta\right) \frac{\omega_{0}}{8 \pi} \tau}}{1+e^{\omega_{0} \beta}}\left[1-e^{\operatorname{coth}\left(\frac{1}{2} \omega_{0} \beta\right) \frac{\omega_{0}}{8 \pi} \tau}\right], \\
\sigma_{-}(\tau)=-\sigma_{0+} e^{-\operatorname{coth}\left(\frac{1}{2} \omega_{0} \beta\right) \frac{\omega_{0}}{8 \pi} \tau}+\frac{e^{-\operatorname{coth}\left(\frac{1}{2} \omega_{0} \beta\right) \frac{\omega_{0}}{8 \pi} \tau}}{1+e^{\omega_{0} \beta}}\left[1+e^{\omega_{o} \beta} e^{\operatorname{coth}\left(\frac{1}{2} \omega_{0} \beta\right) \frac{\omega_{0}}{8 \pi} \tau}\right] .
\end{gathered}
$$

The above equations (96) and (97) exhibit a non-trivial intertwining among $\sigma_{0+}, \beta, \omega_{0}$ that determines the time evolution of the system. Figure 1 depicts two distinct situations. The left curves correspond to the high temperature behaviour of the system, $\omega_{0} \beta \ll 1$; in this case, the system evolves from a state in which $\sigma_{+}(0) \ll \sigma_{-}(0)$, reaching an equilibrium state where both levels are equally filled. At low temperatures, as illustrated by the graph at right for $\omega_{0} \beta \gg 1$, when there are initially many excited particles, $\sigma_{+}(0) \gg \sigma_{-}(0)$, the system evolves in such a way to completely fill $\sigma_{-}$. The intermediate conditions vary between these two extremes. Also, it turns out that the $\beta$ is primarily responsible for determining the values of $\sigma_{ \pm}(\infty)$ for which the system converges, the parameter $\omega_{0}$ giving the rate in which it converges.

Finally, in the limit $\tau \rightarrow \infty$, we obtain in both situations

$$
\begin{gathered}
\sigma_{+}(\infty)=\frac{1}{1+e^{\omega_{0} \beta}}, \\
\sigma_{-}(\infty)=\frac{e^{\omega_{0} \beta}}{1+e^{\omega_{0} \beta}} .
\end{gathered}
$$

We then conclude that the two-level atomic system obeys the Fermi-Dirac statistics, as expected, according to Bloch theorem.

Considering now the uniformly accelerated frame with parametrization (7), we obtain

$$
\begin{aligned}
g\left(\tau^{\prime}, \tau^{\prime \prime}\right) & =\frac{\alpha^{2}}{4 \pi^{2}} \sum_{n=-\infty}^{\infty} \frac{1}{\left|\cosh \left(\alpha \tau^{\prime}\right)-\cosh \left(\alpha \tau^{\prime \prime}\right)\right|^{2}-\left[\sinh \left(\alpha \tau^{\prime}\right)-\sinh \left(\alpha \tau^{\prime \prime}\right)-i \beta \alpha n\right]^{2}} \\
& =\frac{\alpha\left[\operatorname{coth}\left(\frac{\pi\left(e^{\alpha \tau^{\prime}}-e^{\alpha \tau^{\prime \prime}}\right)}{\alpha \beta}\right)-\operatorname{coth}\left(\frac{2 \pi e^{-\frac{1}{2} \alpha\left(\tau^{\prime}+\tau^{\prime \prime}\right)} \sinh \left(\frac{1}{2} \alpha\left(\tau^{\prime}-\tau^{\prime \prime}\right)\right)}{\alpha \beta}\right)\right]}{8 \pi \beta\left[\cosh \left(\alpha \tau^{\prime}\right)-\cosh \left(\alpha \tau^{\prime \prime}\right)\right]} .
\end{aligned}
$$

As in the inertial case, the equation (98) is too complex to be integrated. However, limiting cases provide us remarkable results. Thus, in the limit $\alpha \rightarrow 0$, we obtains

$$
g\left(\tau^{\prime}, \tau^{\prime \prime}\right)=-\frac{1}{4 \beta^{2}} \operatorname{csch}\left(\frac{\pi\left(\tau^{\prime}-\tau^{\prime \prime}\right)}{\beta}\right)^{2}
$$


recovering, as expected, the inertial result and assuring the correctness of $g\left(\tau^{\prime}, \tau^{\prime \prime}\right)$. In the limit of low temperatures, ie, $\beta \rightarrow \infty$, we find

$$
g\left(\tau^{\prime}, \tau^{\prime \prime}\right)=-\frac{\alpha^{2}}{16 \pi^{2}} \operatorname{csch}\left(\frac{\alpha\left(\tau^{\prime}-\tau^{\prime \prime}\right)}{2}\right)^{2}
$$

By comparing (99) with (100), we obtain the relation proposed by Unruh,

$$
T=\frac{\alpha}{2 \pi k_{B}}
$$

i.e. the same result holds both for an accelerated observer at zero temperature as for an inertial observer immersed in a thermal bath at temperature $T$.

Though, looking at the graph in Figure2 to $g\left(\tau^{\prime}, \tau^{\prime \prime}\right)$ we found that the behaviour regarding the temperature and acceleration are not the same. Note that, at zero temperature, as the acceleration increases, $g\left(\tau^{\prime}, \tau^{\prime \prime}\right)$ grows almost linearly. However, fixing a non-zero temperature, results that by increasing the acceleration $g\left(\tau^{\prime}, \tau^{\prime \prime}\right)$ decreases. We conclude that the temperature influences the way the proper acceleration behaves.

\section{FINAL CONSIDERATIONS}

In this paper, after introducing the original motivation behind the so-called Unruh-Fulling-Davies-DeWitt effect through the response function of a detector, which undergoes hyperbolic motion, we have shown that the Thermofield Dynamics (FTD) formalism revealed itself as a natural scenario for describing the behaviour of accelerated systems at finite temperature. In such approach, the thermal states belong to a Hilbert space in the number representation and describe a microscopic system interacting with a reservoir, leading to equations of motion for both the two-level detector and the radiation field, so that in the coarse-graining approach, the dynamics of these systems consist of a Markovian process. Supposing that the sub-systems reach a global thermal equilibrium at a common temperature, we have shown that the local fluctuation due to admissible couplings, enlarging the class of DeWitt detectors, lead to a master equation for the population levels whose dynamics respect the fluctuation-dissipation theorem. As a result, we arrived at the conclusion that the two-point correlation function associated to the population levels depend on both the reservoir equilibrium temperature and the magnitude of the hyperbolic acceleration, indicating the existence of a range of temperatures in which the population of the excited state may also decrease at increasing acceleration magnitudes [26] and, therefore, Unruh and reservoir temperatures do not coincide, although the detailed balance condition is respected. This is corroborated by the analysis of the vacuum fluctuation and radiation reaction hermitian contributions to the energy variation rates of the scalar field considered as a thermal reservoir by choosing a suitable operator ordering. So, we have found that the evolution equation for an accelerated observer coincides with the corresponding one for an inertial observer immersed in a thermal Unruh bath if, and only if, one ascribes a priori a zero value temperature to the radiation field in Rindler coordinates.

\section{Appendix A: The Coarse-Graining approximation}

In section III the operator $\sigma_{A}\left(t^{\prime \prime}\right)$ has been replaced by $\sigma_{A}(t)$, which is equivalent to expanding the master equation

only to second order. If applied recursively, this procedure will generate contributions to higher orders, appearing terms of the type triple, quadruple commutators, and so on. The formal convergence analysis of this series is of extreme complexity, being out of the scope of this paper. However, we present elements of plausibility, which indicate that, at least, the series should asymptotically converge. The exact expression for the population is

$$
\Delta \sigma_{A}(t)=\left(\frac{1}{i \hbar}\right)^{2} \int_{t}^{t+\Delta t} d t^{\prime} \int_{t}^{t^{\prime}} d t^{\prime \prime} \operatorname{Tr}_{R}\left[V\left(t^{\prime}\right),\left[V\left(t^{\prime \prime}\right), \rho\left(t^{\prime \prime}\right)\right]\right]
$$

Integrating the equation for $\rho$ between $t$ and $t^{\prime \prime}$,

$$
\rho\left(t^{\prime \prime}\right)-\rho(t)=\frac{1}{i \hbar} \int_{t}^{t^{\prime \prime}} d t^{\prime \prime \prime}\left[V\left(t^{\prime \prime \prime}\right), \rho\left(t^{\prime \prime \prime}\right)\right]
$$


and substituting in the above equation naturally gives rise to the second and third order terms in the expansion,

$$
\begin{gathered}
\Delta \sigma_{A}(t)=\left(\frac{1}{i \hbar}\right)^{2} \int_{t}^{t+\Delta t} d t^{\prime} \int_{t}^{t^{\prime}} d t^{\prime \prime} \operatorname{Tr}_{R}\left[V\left(t^{\prime}\right),\left[V\left(t^{\prime \prime}\right), \rho(t)\right]\right] \\
+\left(\frac{1}{i \hbar}\right)^{3} \int_{t}^{t+\Delta t} d t^{\prime} \int_{t}^{t^{\prime}} d t^{\prime \prime} \int_{t}^{t^{\prime \prime}} d t^{\prime \prime \prime} \operatorname{Tr}_{R}\left[V\left(t^{\prime}\right),\left[V\left(t^{\prime \prime}\right),\left[V\left(t^{\prime \prime \prime}\right), \rho\left(t^{\prime \prime \prime}\right)\right]\right]\right] .
\end{gathered}
$$

Due to the shape of the potential, we find that the traces can be factored into a part referring to the system $A$ and the other, $g(\tau)$, to the system $R$. This will allow us to ignore the higher order terms. We must show that, after a time interval $\Delta t$ sufficiently large, the three points correlation of the reservoir observable are more strongly suppressed. Projecting on the $R$ basis we have

$$
\begin{gathered}
g\left(t^{\prime}, t^{\prime \prime}\right)=\operatorname{Tr}\left[\sigma_{R} R\left(t^{\prime}-t^{\prime \prime}\right) R\right]=Z_{R}^{-1} \sum_{m, n} e^{-\beta E_{n}}\left\langle n\left|R^{S}\right| m\right\rangle\left\langle m\left|R^{S}\right| n\right\rangle e^{-i \omega_{n m}\left(t^{\prime}-t^{\prime \prime}\right)}, \\
g\left(t^{\prime}, t^{\prime \prime}, t^{\prime \prime \prime}\right)=\operatorname{Tr}\left[\sigma_{R} R\left(t^{\prime}-t^{\prime \prime}\right) R R\left(t^{\prime \prime \prime}-t^{\prime \prime}\right)\right] \\
=Z_{R}^{-1} \sum_{m, n, l} e^{-\beta E_{n}}\left\langle n\left|R^{S}\right| m\right\rangle\left\langle m\left|R^{S}\right| l\right\rangle\left\langle l\left|R^{S}\right| n\right\rangle e^{-i \omega_{n m}\left(t^{\prime}-t^{\prime \prime}\right)} e^{-i \omega_{n l}\left(t^{\prime \prime}-t^{\prime \prime \prime}\right) .}
\end{gathered}
$$

We cannot go further without additional information about the reservoir. However, the reservoir has dense, almost continuous, spectrum of energy so we assume that the different phases in $g\left(t^{\prime}, t^{\prime \prime}\right)$ cause a destructive interference as time increases. Thus, $g\left(t^{\prime}, t^{\prime \prime}\right)$ oscillates very rapidly and its contribution to the integral becomes smaller. Considering two different time scales, such that

$$
T_{A} \gg \Delta t \gg \tau_{c}
$$

where $\tau_{c}$ is the time correlation between observables of the reservoir and $T_{A}$ the time evolution associated with the microscopic system, we have at second order,

$$
\left|\frac{\Delta \sigma_{A}}{\Delta t}\right|^{(2)} \sim \frac{1}{\Delta t} \frac{v^{2}}{\hbar^{2}} \sigma_{A}\left|\int_{t}^{t+\Delta t} d t^{\prime} \int_{t}^{t^{\prime}} d t^{\prime \prime} e^{-i \omega\left(t^{\prime}-t^{\prime \prime}\right)}\right|,
$$

close to a given dominant frequency

$$
\omega_{m n} \equiv \omega \sim \tau_{c}^{-1} .
$$

In the expression, $v \equiv\langle V(\beta)\rangle$ is the typical value of the interaction potential at equilibrium, the observable microscopic system is taken at a given time, around which it does not vary appreciably within the integration intervals. Performing the integral

$$
\begin{gathered}
\int_{t}^{t+\Delta t} d t^{\prime} \int_{t}^{t^{\prime}} d t^{\prime \prime} e^{-i \omega\left(t^{\prime}-t^{\prime \prime}\right)}=\frac{2}{\omega} \int_{t}^{t+\Delta t} d t^{\prime} e^{-i \frac{\omega}{2}\left(t^{\prime}-t\right)} \sin \left[\frac{\omega}{2}\left(t^{\prime}-t\right)\right] \\
\cong \frac{2}{\omega} e^{-i \omega \frac{\Delta t}{4}} \sin \left[\frac{\omega \Delta t}{4}\right] \Delta t
\end{gathered}
$$

therefore,

$$
\left|\frac{\Delta \sigma_{A}}{\Delta t}\right|^{(2)} \sim 2 \tau_{c} \frac{v^{2}}{\hbar^{2}} \sigma_{A} \sim \frac{\sigma_{A}}{T_{R}} \sim \sigma_{A}\left(\frac{\tau_{c}}{T_{R}}\right) \tau_{c}^{-1}
$$

implying

$$
\frac{v^{2}}{\hbar^{2}} \tau_{c}^{2} \ll 1
$$


To third order we have

$$
\left|\frac{\Delta \sigma_{A}}{\Delta t}\right|^{(3)} \sim \frac{1}{\Delta t} \frac{v^{3}}{\hbar^{3}} \sigma_{A}\left|\int_{t}^{t+\Delta t} d t^{\prime} \int_{t}^{t^{\prime}} d t^{\prime \prime} \int_{t}^{t^{\prime \prime}} d t^{\prime \prime \prime} e^{-i \omega\left(t^{\prime}-t^{\prime \prime \prime}\right)}\right|
$$

resulting

$$
\left|\frac{\Delta \sigma_{A}}{\Delta t}\right|^{(3)} \sim 4 \frac{v^{3}}{\hbar^{3}} \tau_{c}^{2} \sigma_{A} \sim\left|\frac{\Delta \sigma_{A}}{\Delta t}\right|^{(2)} 2 \frac{v}{\hbar} \tau_{c} \ll\left|\frac{\Delta \sigma_{A}}{\Delta t}\right|^{(2)} .
$$

Then, in weakly coupled systems, the contribution of higher order terms is less than the lower order ones, even though the series does not converge uniformly, being only asymptotic.

\section{Appendix B: Derivative Coupling}

In this appendix we ask ourselves what could happen if instead of (50), we had a derivative coupling. Thus, we propose a more general interaction given by

$$
H_{I}=\mu R_{2}(\tau) \frac{1}{\omega_{0}^{n}} \frac{d^{n}}{d \tau^{n}} \phi(\tau)
$$

where the constant $\omega_{0}^{n}$ is introduced to ensure the correct dimension. With the interaction (B1), we find new equations for the vacuum fluctuations and radiation reaction, equivalent to the equations (77) and (78),

$$
\begin{gathered}
\left\langle\frac{d}{d \tau} H_{A}(\tau)\right\rangle_{V F}=-\frac{\omega_{0} \mu^{2}}{\omega_{0}^{2 n}} \int_{\tau_{0}}^{\tau} d \tau^{\prime} \cos \left(\omega_{0}\left(\tau-\tau^{\prime}\right)\right)\left\langle a\left|R_{3}^{f}\left(\tau_{0}\right)\right| a\right\rangle \times \\
{\left[i(2 \lambda-1) \frac{d^{n}}{d \tau^{n}} \frac{d^{n}}{d \tau^{\prime n}} \chi^{F}\left(\tau, \tau^{\prime}\right)+\frac{d^{n}}{d \tau^{n}} \frac{d^{n}}{d \tau^{\prime n}} C^{F}\left(\tau, \tau^{\prime}\right)\right]} \\
\left\langle\frac{d}{d \tau} H_{A}(\tau)\right\rangle_{R R}=\frac{\omega_{0} \mu^{2}}{\omega_{0}^{2 n}} \int_{\tau_{0}}^{\tau} d \tau^{\prime}\left[\frac{d^{n}}{d \tau^{n}} \frac{d^{n}}{d \tau^{\prime n}} \chi^{F}\left(\tau, \tau^{\prime}\right)\right] \times \\
{\left[\frac{1}{2} \sin \left(\omega_{0}\left(\tau-\tau^{\prime}\right)\right)+i(2 \lambda-1) \cos \left(\omega_{0}\left(\tau-\tau^{\prime}\right)\right)\left\langle a\left|R_{3}^{f}\left(\tau_{0}\right)\right| a\right\rangle\right] .}
\end{gathered}
$$

Choosing the symmetrical order, $\lambda=\frac{1}{2}$, we eliminate the divergences both in (B2) and (B33). Then, by solving the integrals present in (B2) and (B3), we recover (80) and (81) for the non-derivative case. We thus observe that both results are independent of the coupling order, always giving the same energy variations.

\section{Appendix C: Field Energy Variation}

In section $\amalg I \mathrm{~B}$, we derived an equation for the energy variation rate of the atomic system due to vacuum fluctuations and radiation reaction. In this appendix, we show that the energy balance is respected, observing that the energy variation of the field is equal, in module, to that of the atomic system. We start from the Heisenberg equation (53)

$$
\frac{d}{d \tau} H_{F}(\tau)=i\left[H_{I}(\tau), H_{F}(\tau)\right]=i \mu R_{2}(\tau)\left[\phi(\tau), H_{F}(\tau)\right] .
$$

As previously said, it is advantageous to symmetrizing the operators in the evolution equation,

$$
\begin{aligned}
\frac{d}{d \tau} H_{F}(\tau)=\mu \frac{1}{2}\{ & \left.\left(\partial_{\tau} \phi^{f}(\tau)\right)\left(R_{2}^{f}(\tau)+R_{2}^{S}(\tau)\right)+\left(R_{2}^{f}(\tau)+R_{2}^{S}(\tau)\right) \partial_{\tau} \phi^{f}(\tau)\right\} \\
& +\mu \frac{1}{2}\left\{\left(\partial_{\tau} \phi^{S}(\tau)\right) R_{2}^{f}(\tau)+R_{2}^{f}(\tau) \partial_{\tau} \phi^{S}(\tau)\right\}
\end{aligned}
$$


We separate again the free of the field responsible for the vacuum fluctuation,

$$
\left(\frac{d}{d \tau} H_{F}(\tau)\right)_{V F}=\mu \frac{1}{2}\left\{\left(\partial_{\tau} \phi^{f}(\tau)\right)\left(R_{2}^{f}(\tau)+R_{2}^{S}(\tau)\right)+\left(R_{2}^{f}(\tau)+R_{2}^{S}(\tau)\right) \partial_{\tau} \phi^{f}(\tau)\right\}
$$

as well as the interaction part, responsible for the radiation reaction,

$$
\left(\frac{d}{d \tau} H_{F}(\tau)\right)_{R R}=\mu \frac{1}{2}\left\{\left(\partial_{\tau} \phi^{S}(\tau)\right) R_{2}^{f}(\tau)+R_{2}^{f}(\tau) \partial_{\tau} \phi^{S}(\tau)\right\}
$$

Taking the expectation value in the field vacuum $|0\rangle$ and the atomic state $|a\rangle$, we obtain

$$
\begin{aligned}
& \left\langle\frac{d}{d \tau} H_{F}(\tau)\right\rangle_{V F}=-i \mu^{2} \frac{1}{2} \int_{\tau_{0}}^{\tau} d \tau^{\prime}\left\langle a\left|\left[R_{2}^{f}(\tau), R_{2}^{f}\left(\tau^{\prime}\right)\right]\right| a\right\rangle \partial_{\tau}\left\langle 0\left|\left[\phi^{f}(\tau), \phi^{f}\left(\tau^{\prime}\right)\right]_{+}\right| 0\right\rangle, \\
& \left\langle\frac{d}{d \tau} H_{F}(\tau)\right\rangle_{R R}=-i \mu^{2} \frac{1}{2} \int_{\tau_{0}}^{\tau} d \tau^{\prime}\left\langle a\left|\left[R_{2}^{f}(\tau), R_{2}^{f}\left(\tau^{\prime}\right)\right]_{+}\right| a\right\rangle \partial_{\tau}\left\langle 0\left|\left[\phi^{f}(\tau), \phi^{f}\left(\tau^{\prime}\right)\right]\right| 0\right\rangle,
\end{aligned}
$$

where we can recognize the correlation functions and susceptibility (67), (66), (69) and (70),

$$
\begin{aligned}
& \left\langle\frac{d}{d \tau} H_{F}(\tau)\right\rangle_{V F}=2 \mu^{2} \int_{\tau_{0}}^{\tau} d \tau^{\prime} \chi^{A}\left(\tau, \tau^{\prime}\right) \partial_{\tau} C^{F}\left(\tau, \tau^{\prime}\right), \\
& \left\langle\frac{d}{d \tau} H_{F}(\tau)\right\rangle_{R R}=2 \mu^{2} \int_{\tau_{0}}^{\tau} d \tau^{\prime} C^{A}\left(\tau, \tau^{\prime}\right) \partial_{\tau} \chi^{F}\left(\tau, \tau^{\prime}\right) .
\end{aligned}
$$

Finally, replacing the functions found for $C^{A}, C^{F}, \chi^{A}$ and $\chi^{F}$, for the asymptotic case, $\tau_{0} \rightarrow-\infty$, results

$$
\begin{gathered}
\left\langle\frac{d}{d \tau} H_{F}(\tau)\right\rangle_{V F}=\frac{\mu^{2}}{4 \pi^{2}} \sum_{k=-\infty}^{\infty} \int_{0}^{\infty} d u \sin \left(\omega_{0} u\right)\left[\frac{1}{\left(u-i \epsilon 2+i \frac{2 \pi}{\alpha} k\right)^{3}}+\frac{1}{\left(u+i \epsilon 2+i \frac{2 \pi}{\alpha} k\right)^{3}}\right]\left\langle a\left|R_{3}\right| a\right\rangle, \\
\left\langle\frac{d}{d \tau} H_{F}(\tau)\right\rangle_{R R}=\frac{\mu^{2}}{8 \pi^{2} i} \sum_{k=-\infty}^{\infty} \int_{0}^{\infty} d u \cos \left(\omega_{0} u\right)\left[\frac{1}{\left(u-i \epsilon 2+i \frac{2 \pi}{\alpha} k\right)^{3}}-\frac{1}{\left(u+i \epsilon 2+i \frac{2 \pi}{\alpha} k\right)^{3}}\right]
\end{gathered}
$$

solving the integrals, one finds the same absolute values, obtained in (80) and (81), ensuring that the energy balance is respected. 

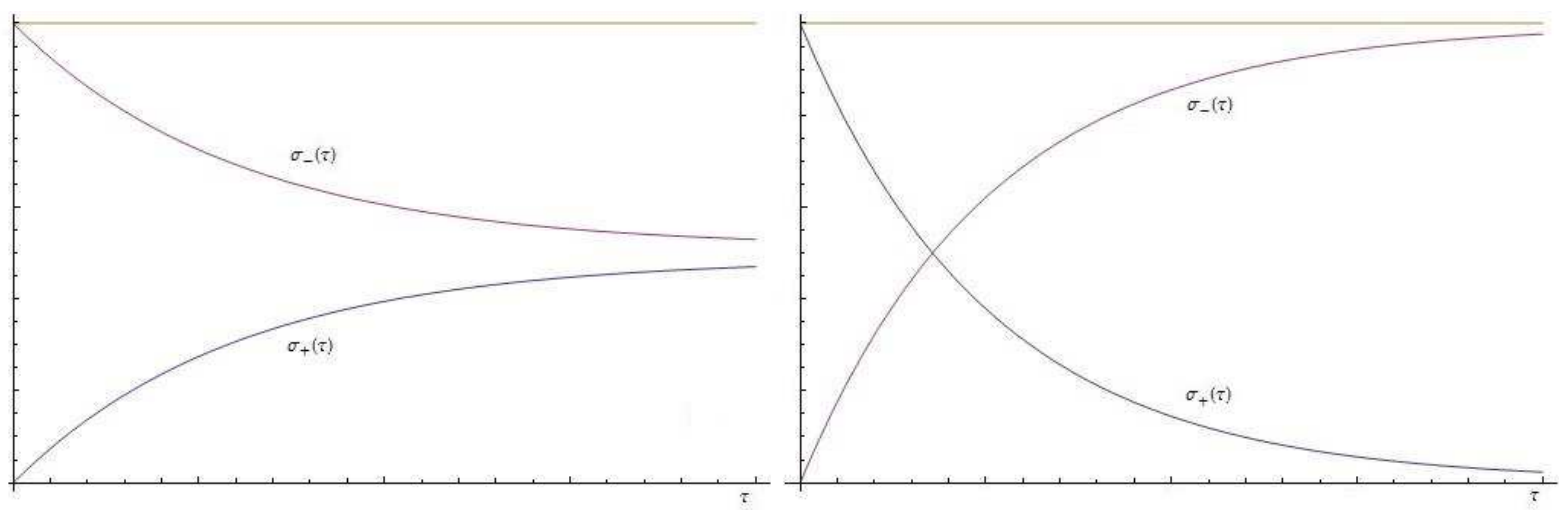

Figure 1. The evolution of $\sigma_{+}(\tau)$ and $\sigma_{-}(\tau)$ at high (left) and low (right) temperature regimes.

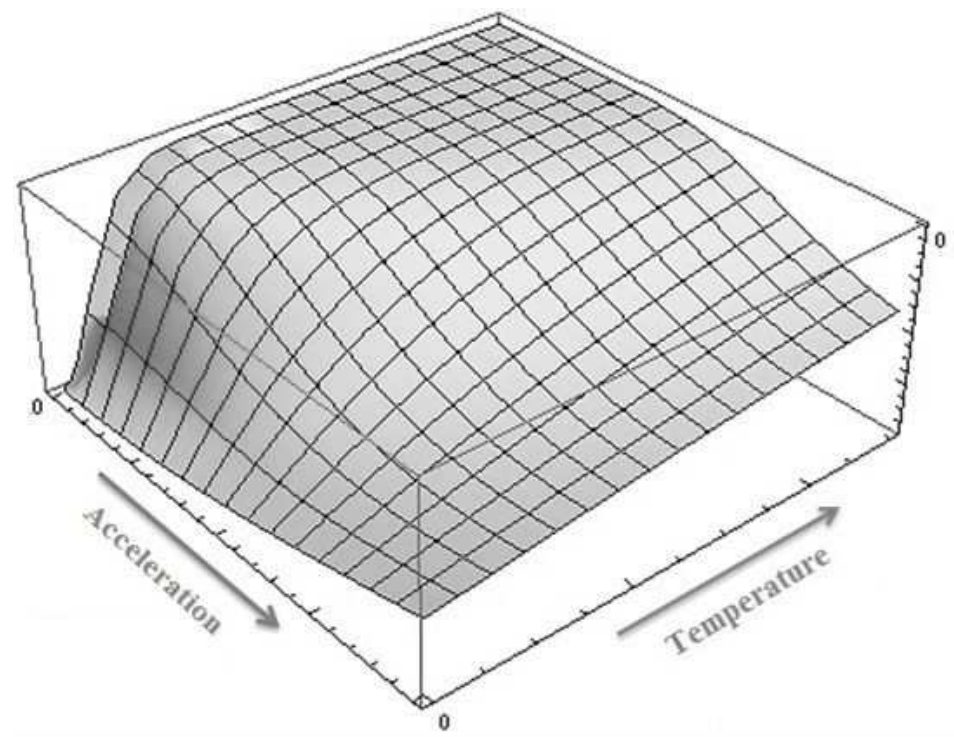

Figure 2. The two-point function $g\left(\tau^{\prime}, \tau^{\prime \prime}\right)$

[1] T. Matsubara, Prog. Theor. Phys. 14, 351 (1955).

[2] J. Schwinger, J. Math. Phys. 2, 407 (1961).

[3] L. Keldysh, Eksp. Teor. Fiz 47, 1515 (1964).

[4] Y. Takahashi and H. Umezawa, Collective Phenomena, 2, 55 (1975), reprinted in Int. J. Mod. Phys. B 10, 1599 (1996).

[5] H. Umezawa, H. Matsumoto, and M. Tachiki, Thermo field dynamics and condensed states (North Holland, 1982).

[6] J. Kapusta and C. Gale, Finite-temperature field theory: Principles and applications (Cambridge Univ Pr, 2006).

[7] A. Das, Finite temperature field theory (World Scientific, 1997).

[8] F. C. Khanna, A. P. C. Malbouisson, J. M. C. Malbouisson, and A. E. Santana, Thermal quantum field theory: algebraic aspects and applications (World Scientific Pub Co Inc, 2009).

[9] J. L. Tomazelli and L. C. Costa, Int. J. Mod. Phys. A 18, 1079 (2003).

[10] H. Umezawa, Advanced field theory: micro, macro, and thermal physics (Amer Inst of Physics, 1995).

[11] S. Hawking, Commun. Math. Phys. 43, 199 (1975)

[12] S. W. Hawking, Nature 248, 30 (1974).

[13] P. C. W. Davies, J. Phys. A: Math. Gen. 8, 609 (1975)

[14] W. G. Unruh, Phys. Rev. D 14, 870 (1976)

[15] P. C. W. Davies, Reports on Progress in Physics 41, 1313 (1978)

[16] D. Sciama, P. Candelas, and D. Deutsch, Adv. Phys. 30, 327 (1981)

[17] N. D. Birrell and P. C. W. Davies, Quantum fields in curved space (Cambridge University Press, 1982). 
[18] B. DeWitt, Phys. Rep. 19, 295 (1975).

19] J. Audretsch and R. Müller, Phys. Rev. D 49, 4056 (1994).

[20] J. Audretsch and R. Müller, Phys. Rev. A 52, 629 (1995).

[21] C. Cohen-Tannoudji, J. Dupont-Roc, and G. Grynberg, Atom-photon interactions: basic processes and applications (Wiley, 1992).

[22] G. W. Ford, J. T. Lewis, and R. F. O'Connell, Phys. Rev. A 37, 4419 (1988)

[23] J. J. Halliwell and T. Yu, Phys. Rev. D 53, 2012 (1996).

[24] G. W. Ford and R. F. O'Connell, Phys. Rev. D 64, 105020 (2001)

[25] B. L. Hu, J. P. Paz, and Y. Zhang, Phys. Rev. D 45, 2843 (1992)

[26] W. G. Brenna, R. B. Mann, and E. Martin-Martinez, ArXiv e-prints (2015), arXiv:1504.02468. 